\title{
All-Dielectric Terahertz Half-Wave Plate with Antireflection Layer
}

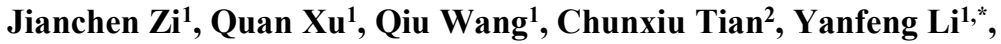 \\ Xixiang Zhang ${ }^{2}$, Jiaguang Han', and Weili Zhang, ${ }^{1,3}$ \\ ${ }^{1}$ Center for Terahertz Waves, College of Precision Instrument and Optoelectronics Engineering, Tianjin University, Key Laboratory \\ of Optoelectronic Information Technology (Ministry of Education of China), Tianjin 300072, People's Republic of China \\ ${ }^{2}$ Division of Physical Science and Engineering, King Abdullah University of Science and Technology, Thuwal 23955-6900, Saudi Arabia \\ ${ }^{3}$ School of Electrical and Computer Engineering, Oklahoma State University, Stillwater, Oklahoma 74078, USA \\ *Corresponding author: yanfengli@tju.edu.cn
}

\begin{abstract}
We present an all-dielectric terahertz half-wave plate with an antireflection layer. The device is made of pure silicon, and can realize cross polarization conversion with almost $100 \%$ conversion rate and $90 \%$ of transmission at the operating frequency.

OCIS codes: (160.3918) Metamaterials; (300.6495) Spectroscopy, terahertz; (260.1440) Birefringence; (260.5430) Polarization.
\end{abstract}

\section{Introduction}

Terahertz (THz) wave is the electromagnetic (EM) wave within the frequency band of 0.1-10 THz. THz technology has seen a rapid development in recent years, and has been applied widely in such diverse fields as communications [1], imaging [2], and spectroscopy [3]. Half-wave plates (HWPs) are a basic device for EM wave manipulation, and as such are also very important for $\mathrm{THz}$ technology, in which efficient devices of various kinds are needed. Nevertheless, the performances of the HWPs reported so far cannot satisfy this demand, and higher performance are desirable. Here, an all-dielectric terahertz HWP made of pure silicon is presented, simulation and experiment have been done, and the results demonstrate its high performance at the operating frequency.

\section{Device Structure}

The all-dielectric terahertz HWP designed here is made of pure silicon, and it comprises two working layers with subwavelength periodic structures. One works as the half-wave-plate layer (HWPL), and the other functions as the antireflection layer (ARL). The unit cells of the two layers are shown in Fig. 1.

As shown in Fig. 1(a), the HWPL is composed of an array of elliptical silicon pillars. The long axis is at an angle of $45^{\circ}$ relative to the $x$ axis. Birefringence is realized in the $u$ and $v$ axes due to the anisotropy of the elliptical pillars, similar to a traditional HWP, and the operating frequency is determined by the structure parameters. With the appropriate parameters, the $x$-polarized incidence along the $z$ axis will be converted into $y$-polarized output at the specific frequency.

The ARL consists of an array of square pillars as shown in Fig. 1(b). Motamedi et al. [4] have demonstrated the antireflection effect by such a structure. It can be used to improve transmission, and its operating frequency also depends on the corresponding structure parameters.
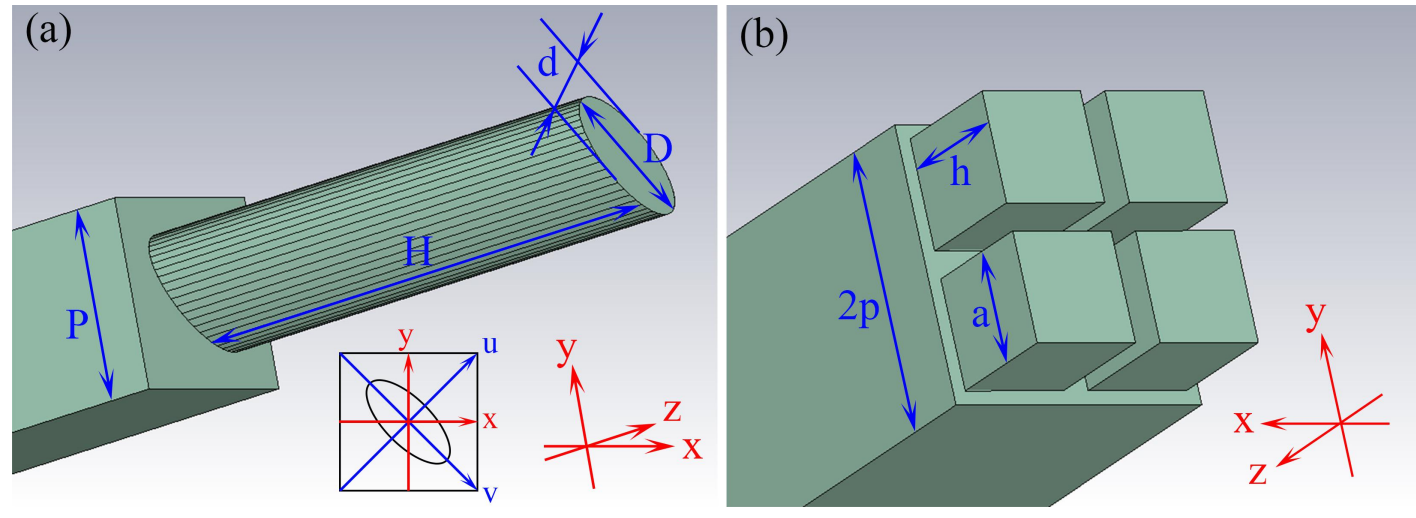

Fig. 1. Unit cell of (a) HWPL and (b) ARL of the THz HWP 


\section{Simulation and Experiment Results}

Numerical simulations have been done with CST Microwave Studio. The structure parameters have been optimized to have $P=2 p=100 \mu \mathrm{m}, H=300 \mu \mathrm{m}, D=80 \mu \mathrm{m}, d=35 \mu \mathrm{m}, h=45 \mu \mathrm{m}$, and $a=40 \mu \mathrm{m}$. The incidence is $x$-polarized THz wave along the $z$ axis. As the results in Fig. 2 (a) show, the HWP designed can realize a conversion of cross polarization for $x$-polarized incidence. The transmission $t_{x y}$ for cross polarization can reach $90 \%$ at $1 \mathrm{THz}$, and meanwhile the polarization conversion rate (PCR) can reach almost $100 \%$. Based on the simulation, a sample was fabricated, and the actual structure parameters were measured to be: $P=104.9 \mu \mathrm{m}, p=52.4 \mu \mathrm{m}, H=285.6 \mu \mathrm{m}, D=92.8$ $\mu \mathrm{m}, d=42.1 \mu \mathrm{m}, h=65.9 \mu \mathrm{m}$, and $a=41.6 \mu \mathrm{m}$. The corresponding results from measurement are shown in Fig. 2 (b), where we can see that the sample could realize the expected polarization conversion at $1.07 \mathrm{THz}$ with a transmission $t_{x y}$ of $80 \%$ and PCR of almost $100 \%$. The discrepancies from design are attributed to the deviation in the sample parameters, where the antireflection effect is more susceptible to structural variation.

(a)

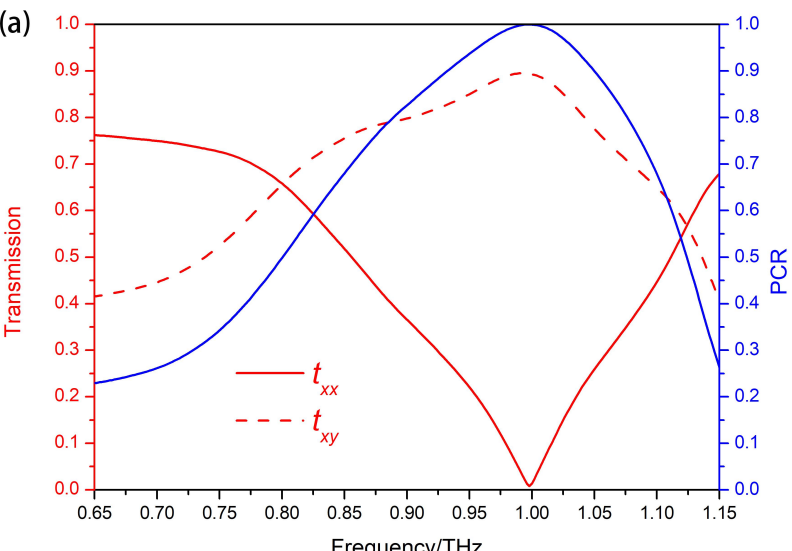

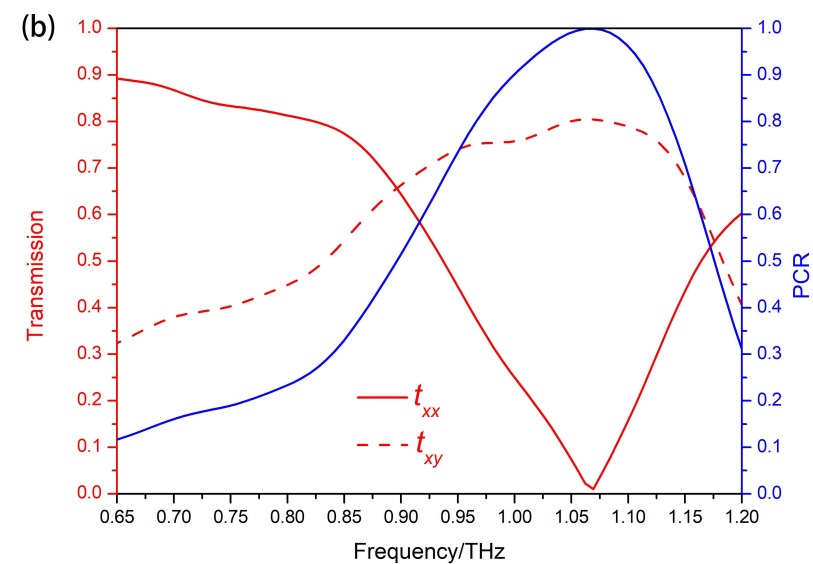

Frequency/THz

Fig. 2. (a) Simulation and (b) experiment results

\section{Conclusion}

We present an all-dielectric terahertz HWP with an ARL. It is made of pure silicon, both simulation and experiment results show that it can achieve a high performance conversion for cross polarization at the designed operating frequency.

\section{Acknowledgments}

This work was supported by the National Basic Research Program of China (2014CB339800), the National Natural Science Foundation of China (61422509, 61622505, 61427814, 61377047 and 61420106006), the Program for Changjiang Scholars and Innovative Research Team in University (IRT13033), the Cooperative Innovation Center of Terahertz Science, and the U.S. National Science foundation (ECCS-1232081).

\section{References}

[1] T. Nagatsuma, G. Ducournau, and C. C. Renaud. "Advances in terahertz communications accelerated by photonics." Nature Photonics 10 , 371-379 (2016).

[2] S. Katletz, et al. "Polarization sensitive terahertz imaging: detection of birefringence and optical axis." Optics Express 20, 23025-23035 (2012).

[3] P. U. Jepsen, D. G. Cooke, and M. Koch. "Terahertz spectroscopy and imaging - Modern techniques and applications." Laser \& Photonics Reviews 5, 124-166 (2011).

[4] M. E. Motamedi, W. H. Southwell, and W. J. Gunning. "Antireflection surfaces in silicon using binary optics technology." Applied Optics 31, 4371-4376 (1992). 